\title{
EVOLUTION OF LONGITUDINAL MODULATION IN ELECTRON BEAMS
}

\author{
C. P. Neuman*, Department of Physics, Queensborough Community College, City University of \\ New York, Bayside, NY, U.S.A. \\ P. G. O'Shea, Department of Electrical and Computer Engineering, University of Maryland, College \\ Park, MD 20742, U.S.A.
}

\begin{abstract}
Electron beams with periodic longitudinal density modulation may produce terahertz radiation in linear accelerators. In this study, simulations are used to understand how the density and energy modulations of an electron bunch evolve as the beam is transported through a linac system. It is instructive to simulate beams that have only density modulation and beams that have only energy modulation. The former is useful for learning how to keep the desired density modulation for beams intended to create terahertz radiation, the latter for learning how to suppress unwanted energy modulation, which may have originated as density modulation. The simulations in this study are performed using PARMELA in conujnction with other software codes. The study investigates energy ranges that are higher than those studied in the authors' previous work. Parameters in the simulations are chosen to correspond to existing accelerator systems so that the results may be used to support an experimental study.
\end{abstract}

\section{INTRODUCTION}

The purpose of the study is to simulate electron beams with longitudinal modulation. Such beams with subpicosecond features may be used to produce terahertz radiation. In addition, beams may unintentionally have sub-picosecond features that may produce unwanted terahertz radiation. It is important to understand how the features in these beams evolve as the beam is accelerated in a linac. Typical electron beams that produce terahertz radiation have both density and energy modulation in the longitudinal direction. In this study, simulations involve beams that have only density modulation and beams that have only energy modulation, and the effects of each are examined. Separating the types of modulation facilitates a better understanding of how a modulated beam evolves. In addition, if the evolution of a modulated beam can be understood, it may be possible to "clean" a beam that is producing unwanted terahertz radiation.

\section{METHOD OF STUDY}

PARMELA is used to simulate electron beams accelerated through a SLAC-type linear accelerator. The accelerator model used is that of the DUV-FEL at the Source Development $\mathrm{Lab}$ at Brookhaven National Laboratory. An ATF 1.6-cell photoinjecting RF electron gun accelerates electrons to approximately $4 \mathrm{MeV}$, and two linac tanks, each $3 \mathrm{~m}$ long, accelerate the electrons to

\footnotetext{
*cneuman@qcc.cuny.edu
}

approximately $33 \mathrm{MeV}$ and $65 \mathrm{MeV}$, respectively. A charge of $20 \mathrm{pC}$ per electron bunch is used in these simulations. Thus, space charge effects, which cause modulations to wash out, are not significant in this study. A schematic of the accelerator system is shown in Figure 1.

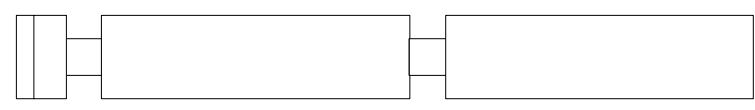

$\mathrm{e}^{-}$gun $\quad$ linac tank $1 \quad$ linac tank 2

Figure 1: Accelerator system for this study.

To create the beams, PARMELA is run up to specified points, such as the end of the gun or tank 1 . The data for the beam is then extracted, processed, modified, and then reinserted into the simulation the same place and with the same phase.

Initially a beam that has a gaussian density profile and flat energy profile at the cathode is run through the gun using PARMELA. The beam data at this point is extracted and analyzed. From the information gathered, a newly created beam could be given a gaussian or a modulated density profile. The modulated shape is a gaussian shape with a sinusoidal variation. The depth of modulation and the period can be varied. The energy profile of the new beam is created by using a sixth-order polynomial curve fit of the original energy profile of the actual energy profile from the simulation. An energy modulated beam is created by superimposing a sinusoidal variation on this fitted curve. Using a curve fit for the energy profile rather than the actual profile is useful because it allows one to perceive more clearly any changes in the energy profile. However, as discussed below, it may not be the most appropriate representation of the energy profile of the beam in some cases. The beam is populated using the desired probablity distribution and is reinserted into the simulation.

It was determined that 50,000 particles are enough to produce accurate results in this simulation. Since the beams are axially symmetric, it is appropriate to use PARMELA's space charge model that assumes annular rings of charge. Test simulations were also run with PARMELA's other space charge calculation which uses a 3-D PIC routine. The results of both space charge models were found to agree, but the latter calculation required significantly more particles. Computing time is proportional to the number of particles in the simulation [1]. Therefore the former space charge model is used for this study. 
Two types of beams are studied: 1) Beams that are density modulated but not energy modulated, and 2) beams that are energy modulated but not density modulated. These beams are not physically realizable, and the simulations give insight into the evolution of modulations that could not be obtained through physical experimentation.

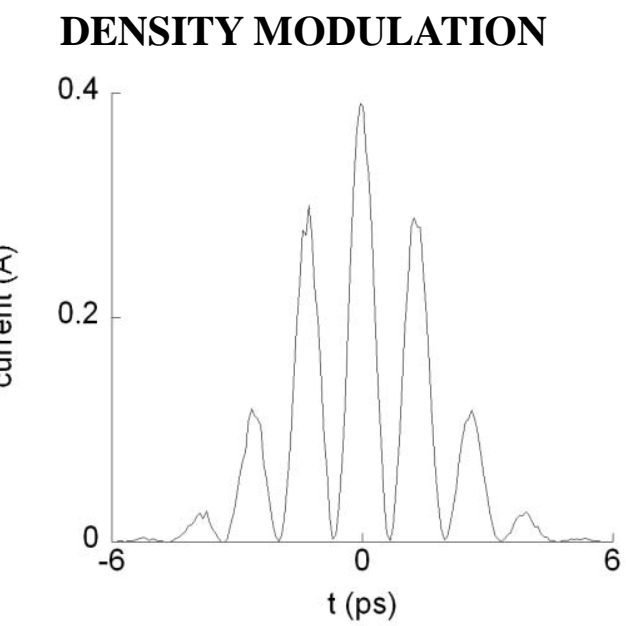

Figure 2: Longitudinal density profile of a density modulated beam.
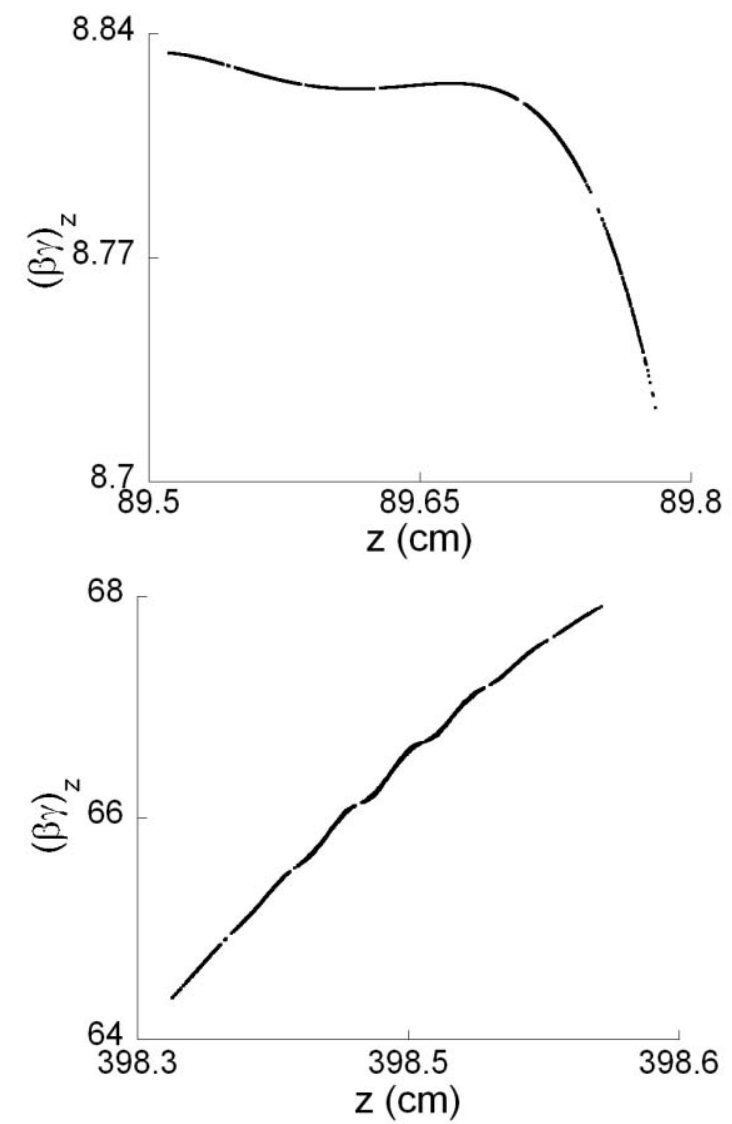

Figure 3: Longitudinal energy profile in the electron beam at the end of the gun (top) and at the end of tank 1 (bottom). Note the modulations in the bottom plot.
An example of a density modulated beam is shown in Figure 2. As reported previously [2, 3], a density modulated beam retains its density modulation through transport in a linac system. It was also shown [2] that a density modulated beam induces energy modulation in the beam, as seen in Figure 3.

To investigate the behavior of density modulated beams for higher energy, modified beams are inserted into the simulation after tank 1 , where the beam energy is $34 \mathrm{MeV}$, and the beam is analyzed at the end of tank 2 . The result is that, unlike the lower energy case studied previously, there is no noticeable change in the energy distribution of the beam. That is, the energy distribution is less malleable at higher energies. The density profile also shows no change.

\section{ENERGY MODULATION}
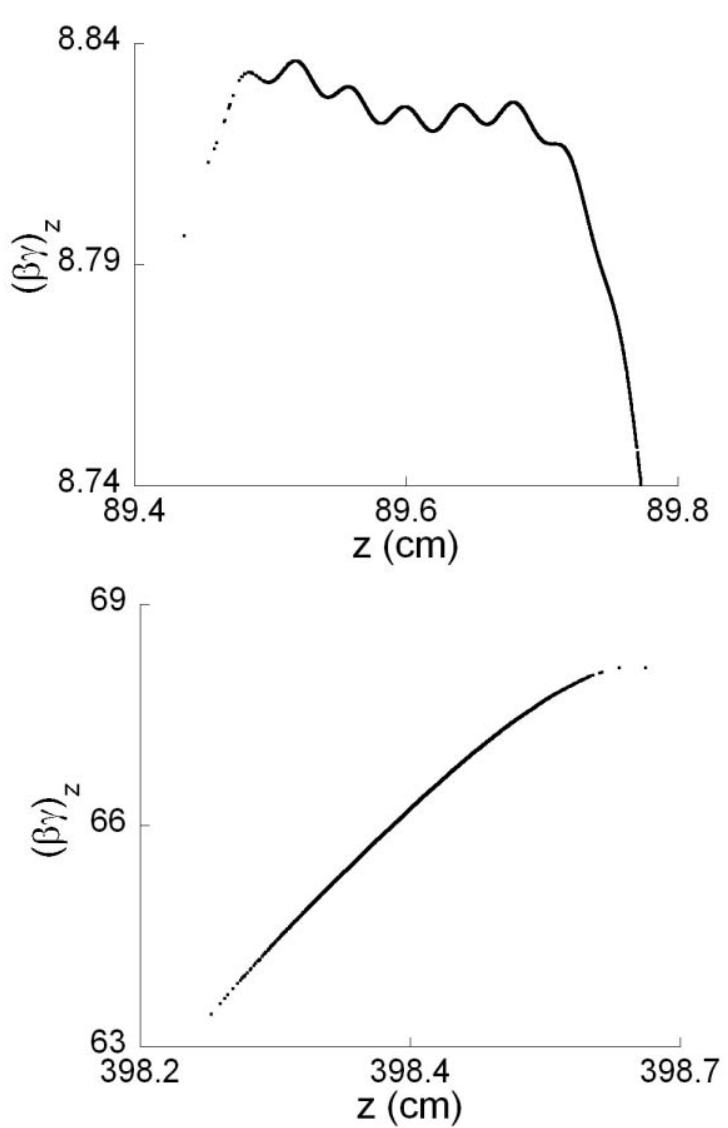

Figure 4: Longitudinal energy profile in the electron beam at the gun (top) and at the end of tank 1 (bottom). The energy modulation washes out in this lower energy case.

For a beam with energy modulation but no density modulation, previous results [2] showed that the energy modulation washes out as the beam is transported through the accelerator system, as shown in Figure 4.

To study higher energy behavior of energy modulated beams, the modified beams are inserted into the simulation at the end of tank 1, where the energy is 34 $\mathrm{MeV}$, and are analyzed at the end of tank 2 . The result is that the energy modulation retains its character but decreases in amplitude, as seen in Figure 5. As in the case

D01 Beam Optics - Lattices, Correction Schemes, Transport 
of a density modulated beam, the energy distribution of an energy modulated beam is less malleable at higher energies.
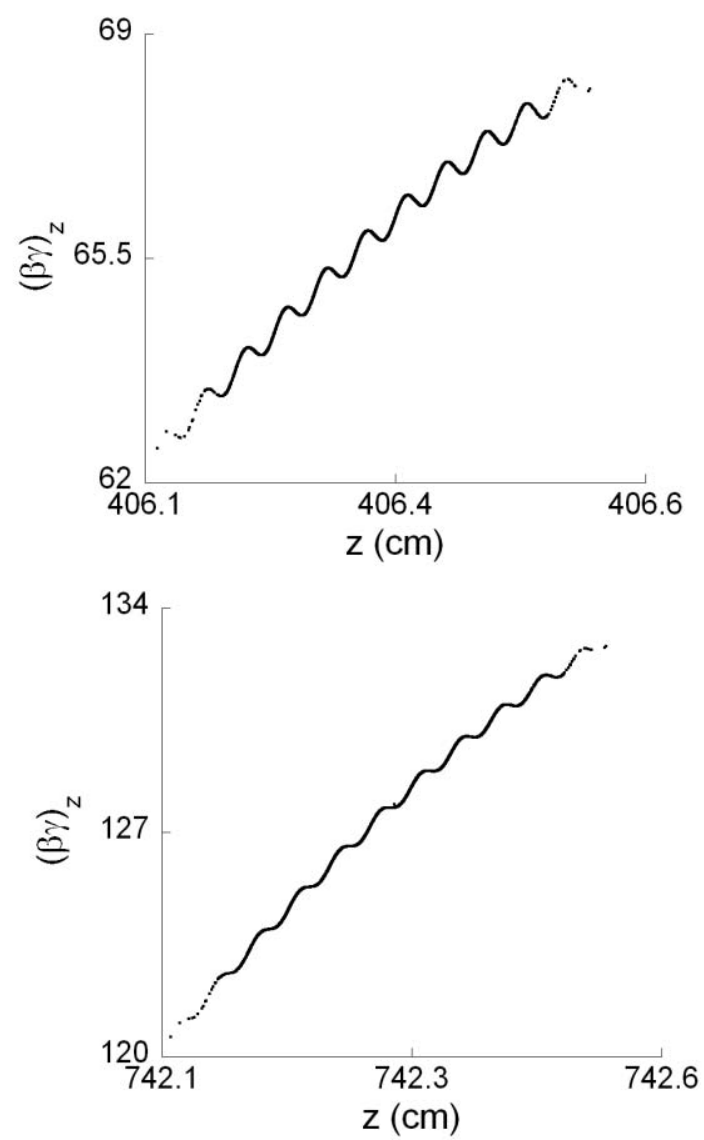

Figure 5: Longitudinal energy profile in the electron beam at the end of tank 1 (top) and at the end of tank 2 (bottom). The energy modulation washes out in this lower energy case.

\section{CONCLUSIONS}

Previous work showed that for lower energies, density modulation remains in the beam during transport through an accelerator system, and that energy modulation diminishes in amplitude. These studies examined beams that were inserted into the simulation just after the gun, at $4 \mathrm{MeV}$. The current study shows that for higher energies, neither density nor energy modulation change while the beam is accelerated. This study examines beams that are inserted into the simulation just after the first accelerator tank, at $34 \mathrm{MeV}$. Thus, the features of a beam are "frozen" into the beam at higher energies.

For cases where longitudinal modulation is required in a beam, such as in a seeded-beam FEL or a terahertz radiation source, these conclusions show that once the beam has the required modulation at high energy, the modulation will remain in the beam.

For cases where the sub-picosecond features of the beam produce unwanted terahertz radiation, these conclusions show that as the energy of the beam increases, it becomes more difficult to alter the beam's density or energy profile. Thus, efforts to "clean" a beam, such as by using a wiggler to convert density modulation into energy modulation [4], will be more successful at lower energies.

It is interesting to point out that while previous work showed that an energy modulated beam loses its modulation at lower energies and does not induce density modulation in the beam, current work [4] shows that an energy modulated beam can induce density modulation in the beam. In the former cases, the energy modulation is created by superimposing a sinusoidal modulation onto the energy profile that was created by a curve fit of beam data from a PARMELA simulation (see Figure 4, top). In the latter case, the energy modulation is created by a wiggler, which converts density modulation into energy modulation. Thus, the latter case involves a more appropriate shape to use for an energy modulated beam. Future work will investigate ways to quantify this profile and use it in future studies.

\section{FUTURE WORK}

Future work will focus on a better model for an energy modulated beam, as discussed above. In addition, future studies will involve simulations that take into account the radiation of the beam, which can affect the beam profile. Finally, other future studies will focus on the evolution of modulations in the electron gun itself. For cases where modulations are created by applying laser pulses to the photocathode, the beam begins with density modulation but no energy modulation. It will be useful to investigate how the modulations evolve inside the electron gun, to better preserve wanted features of the beam and possibly eliminate unwanted features.

\section{ACKNOWLEDGEMENTS}

Thanks to Queensborough Community College and the City University of New York for some support for this study.

\section{REFERENCES}

[1] J. Billen, "PARMELA User Manual", Los Alamos, Los Alamos Accelerator Code Group of Los Alamos National Laboratory (2005).

[2] C.P. Neuman and P.G. O'Shea. Simulation of Longitudinally Modulated Electron Beams. In 2006 Advanced Accelerator Concepts Workshop, AIP Conference Proceedings, 877. Melville, AIP (2006) 621-627.

[3] J. G. Neumann, et al, "Simulations and Experiments of Electron Beams Pre-Modulated at the Photocathode" in Proceedings of the 2005 Particle Accelerator Conference, edited by C. Horak, Piscataway, IEEE (2005).

[4] C. P. Neuman and P. G. O'Shea. "Suppression of Terahertz Radiation in Electron Beams with Longitudinal Density Modulation", these proceedings. 